\title{
Diametral Reachable Index (DRI) of a Vertex
}

\author{
H. B. Walikar \\ Professor \\ Dept of Computer Science, \\ Karnatak University, \\ Dharwad, India.
}

\author{
Shreedevi V. Shindhe \\ Research Scholar \\ Dept of Computer Science \\ Karnatak University, \\ Dharwad, India
}

\begin{abstract}
:
Every graph has one or more diametral paths. A diametral path of a graph is a shortest path whose length is equal to the diameter of the graph. Let $d v$ be a diametral vertex. There may be one or more diametral paths originating from $d v$. We want to find all the diametral paths, originating from $d v$. The total number of diametral paths reachable from a vertex $v$ is called the Diametral Reachable Index of that vertex, denoted $D R I(v)$. For any vertex $v$, the $D R I(v)=0$, if there are no diametral paths reachable from $v$, else we write $D R I(v)=t$, where $t$ is the total number of diametral paths reachable from vertex $v$. An algorithm is developed to find DRI of each vertex of a graph, by modifying the DFS algorithm.
\end{abstract}

Keywords: DRI, diametral paths, diametral reachable index, diametral vertex.

\section{INTRODUCTION}

\subsection{Graph Terminologies:}

In this section the required basic definitions are given $[1$, 2, 3, 4, and 5].

1.1.1 Graph: A graph $G=(V, E)$ is an ordered pair of sets. Elements of $\mathrm{V}$ are called vertices or nodes, and elements of $E \subseteq V \times V$ are called edges or lines. We refer to $V$ as the vertex set of $G$, with $E$ being the edge set.

\subsubsection{Walks, trails, paths, and connected graphs:}

If $u$ and $v$ are two vertices in a graph G, a $u-v$ walk is an alternating sequence of vertices and edges starting with $u$ and ending at $v$. Consecutive vertices and edges are incident. For the graph in Figure 1, an example of a walk is an a - e walk: $a, b, c, b, e$. In other words, we start at vertex a and travel to vertex b. From b, we go to $c$ and then back to $b$ again. Then we end our journey at $e$. Notice that consecutive vertices in a walk are adjacent to each other. One can think of vertices as destinations and edges as footpaths, say. We are allowed to have repeated vertices and edges in a walk. The number of edges in a walk is called its length. For instance, the walk $a, b, c, b, e$ has length 4 .

A trail is a walk with no repeating edges. For example, the $a-b$ walk $a, b, c, d, f, g, b$ in Figure 1 is a trail. It does not contain any repeated edges, but it contains one repeated vertex, i.e. $b$. Nothing in the definition of a trail restricts a trail from having repeated vertices. Where the start and end vertices of a trail are the same, we say that the trail is a circuit,

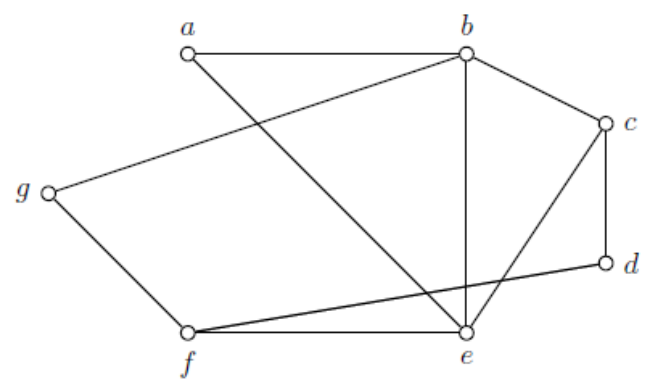

Figure 1: Walking along a graph.

otherwise known as a closed trail. Thus the walk $a, b, e, a$ is a circuit. A walk with no repeating vertices is called a path. Without any repeating vertices, a path cannot have repeating edges; hence a path is also a trail. A path whose start and end vertices are the same is called a cycle. For example, the walk $a, b, c, e, a$ in Figure is a path and a cycle.

A graph is said to be connected if for every pair of distinct vertices $u, v$ there is a $u-v$ path joining them. A graph that is not connected is referred to as disconnected. The empty graph is disconnected and so is any non-empty graph with an isolated vertex.

A geodesic path or shortest path between two distinct vertices $\mathrm{u}, \mathrm{v}$ of a graph is a $u-v$ path of minimum length. A nonempty graph may have several shortest paths between some distinct pair of vertices. For the graph in Figure 1, both $a, b, c$ and $a, e, c$ are geodesic paths between $a$ and $c$.

\subsubsection{Distance in graphs:}

The distance $d(u, v)$ between two (not necessary distinct) vertices $u$ and $v$ in a graph $G$ is the length of a shortest path between them. The subscript $G$ is usually dropped when there is no danger of confusion. When $\mathrm{u}$ and $\mathrm{v}$ are identical, their distance is 0 . When $u$ and $v$ are unreachable from each other, their distance is defined to be infinity $\infty$. The eccentricity $\varepsilon(v)$ of a vertex $v$ in a graph $G$ is the maximum distance from $v$ to any other vertex. $e(v)=$ $\max \{d(u, v): u \in V\}$. 
The diameter $\operatorname{diam}(G)$ of a graph $G$ is the maximum eccentricity over all vertices in a graph; and the radius $\operatorname{rad}(G)$, the minimum. When there are two components in $G, \operatorname{diam}(G)$ and $\operatorname{rad}(G)$ defined to be infinity $\infty$. Trivially, $\operatorname{diam}(G) \leq$ $2 \operatorname{rad}(G)$. Vertices with maximum eccentricity are called peripheral vertices. Vertices of minimum eccentricity form the center. A tree has at most two center vertices.

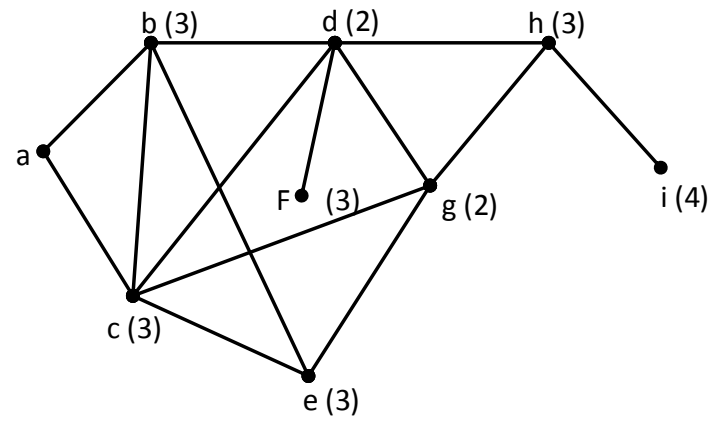

Fig 2: A graph and its eccentricities.

The vertices whose value is equal to maximum eccentricity are called diametral vertices. The paths originating from diametral vertices, whose length is equal to diameter are called diametral paths.

\section{DIAMETRAL REACHABLE INDEX (DRI) OF A VERTEX:}

Every graph has one or more diametral path/s. A diametral path $(D P)$ of a graph is a shortest path whose length is equal to the diameter of the graph.

$$
D P=\operatorname{diam}(G)=\operatorname{dist}(s, d)
$$

Let $d v$ be a diametral vertex. There may be one or more diametral paths originating from $d v$. We want to find all the diametral paths, originating from $d v$. The total number of diametral paths reachable from a vertex $v$ is called the Diametral Reachable Index of that vertex, denoted DRI(v).

For any vertex $v$, the $\operatorname{DRI}(v)=0$, if there are no diametral paths reachable from $v$, else we write $D R I(v)=t$, where $t$ is the total number of diametral paths reachable from vertex $v$. In other words, the DRI of each vertex gives the maximum number of diametral paths reachable from that vertex.

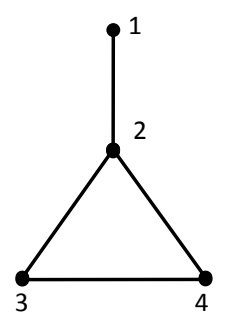

Figure 3(a)

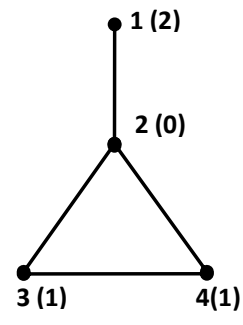

Figure 3(b)
Example 1: The diameter of the graph in fig. 3(a) is 2. The diametral reachable index of each vertex is given in fig 3.(b).

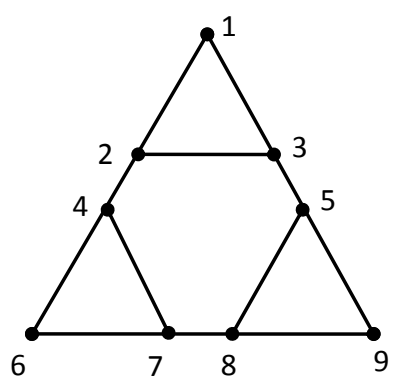

Figure 4 (a)

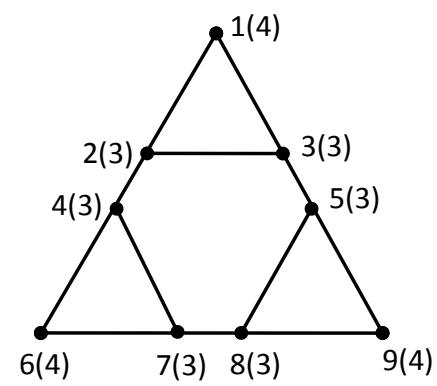

Figure 4 (b)

Example 2: The diameter of the graph in fig. 4(a) is 3. The diametral reachable index of each vertex is given in fig 4.(b).

\section{DRI OF A VERTEX FOR SOME BASIC CLASSES OF GRAPHS:}

\subsection{Complete Graph:}

Let $G$ be a complete graph then $\operatorname{DRI}(v)=n-1$ for all $v \in$ $V$. All the edges of a complete graph are diametral paths. The degree of each vertex is $n-1$. Therefore there are $n-1$ diametral paths reachable from each vertex. Removal of an edge $(u, v)$ from the given complete graph increases the diameter by 1 and $\operatorname{DRI}(u)=\operatorname{DRI}(v)=n-2$ and for all the other vertices $D R I=0$. The example can be seen in figure 5 .
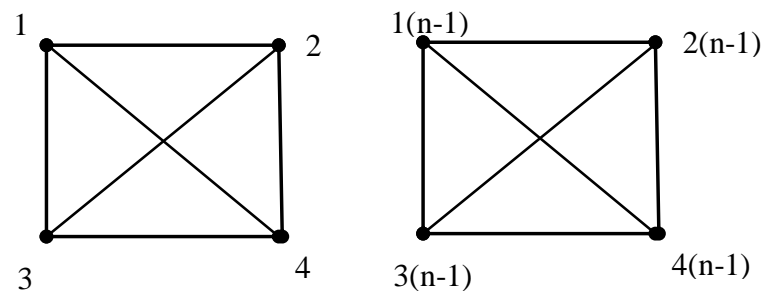

Figure 5(a) : $K_{4}$

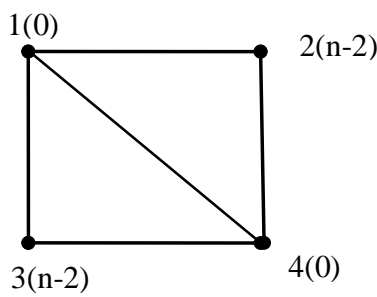

Figure 5: (a) DRI of vertices of a complete graph (b) DRI of vertices of $K_{4}-e$

Figure 5(b) : $K_{4}-e$ 


\subsection{Paths:}

For any graph which is a path, DRI for end vertices will be equal to 1 and for other vertices on the path the DRI is equal to 0 .

The path itself is the only diametral path, and there are no other diametral paths reachable from any vertex of the path, except the end vertices of the path. Therefore, it can be represented collectively as

$$
\operatorname{DRI}\left(v_{1}, v_{2}, \ldots, v_{n}\right)=(1,0,0, \ldots, 0,0,1) .
$$

Example: For $P_{5}$

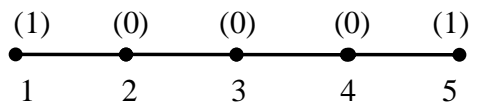

Figure 6: DRI of vertices of a path graph $\boldsymbol{P}_{5}$

\subsection{Star Graphs:}

Let $G$ be a star graph with $k$ spokes ( $n=k+1$ vertices), then DRI for each spoke equals $k-1$ and the central vertex has $D R I=0$. Therefore we can write it $\operatorname{DRI}\left(v_{1}, v_{2}, \ldots, v_{n}\right)=$ $(0, k-1, k-1, \ldots, k-1)$. Example is in figure 7 .

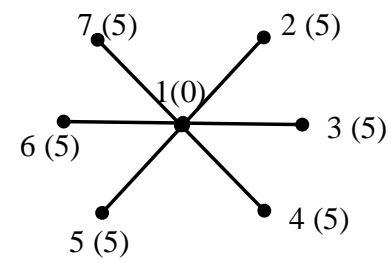

Figure 7: DRI of vertices of a star graph with $k=6$.

\subsection{Caterpillar:}

For any caterpillar, the diametral path consists of the end vertices that have the maximum eccentricity. Using such vertices we can directly find out the DRI of vertices by using a simple algorithm.

Example: Figure 8.

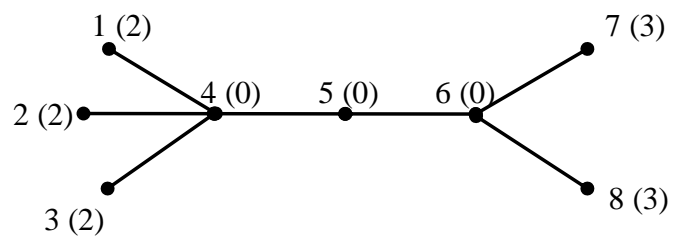

Figure 8: DRI of vertices of a caterpillar.

The algorithm is based on the observation that the end vertices can be divided into two sets based on there adjacency. That is, one set of the eccentric vertices will be adjacent to a common vertex $S 1$ and the other set to another vertex $S 2$. $D R I($ set 1 vertices $)=$ degree of $S 2-1$ and $D R I($ set 2 vertices $)=$ degree of $S 1-1$. i. Find the eccentric vertices of the caterpillar and add to the set $E$.

ii. $\quad$ Find $S 1$ and $S 2$ vertices and their degree

For each eccentric vertex of $E$ find the adjacent vertex $u$

If $u$ is adjacent to $S 1$ then \{

Add $u$ to the set $E 1$

degree $[S 1]++$;

\}

If $u$ is adjacent to $S 2$ then

Add $u$ to the set $E 2$

degree $[S 2]++$;

\}

iii. For each vertex of the set $E 1$ assign $D R I(v)=$ count [S2].

iv. For each vertex of the ser $E 2$ assign $\operatorname{DRI}(v)=$ count [S1].

\subsection{Cycles:}

For any cycle $C_{n}$, the DRI of each vertex is 2 . For cycles the diametral path is $n / 2$. From every vertex there will be two diametral paths.

Example:

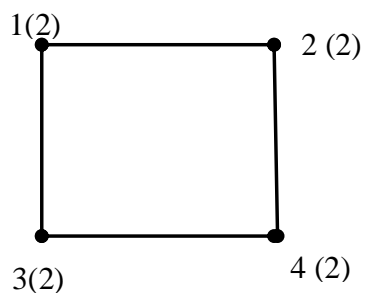

Figure 9: DRI of vertices of cycle $C_{4}$.

\subsection{Petersen Graph:}

For Petersen graph DRI of Each vertex is 6.

Example: figure 10.

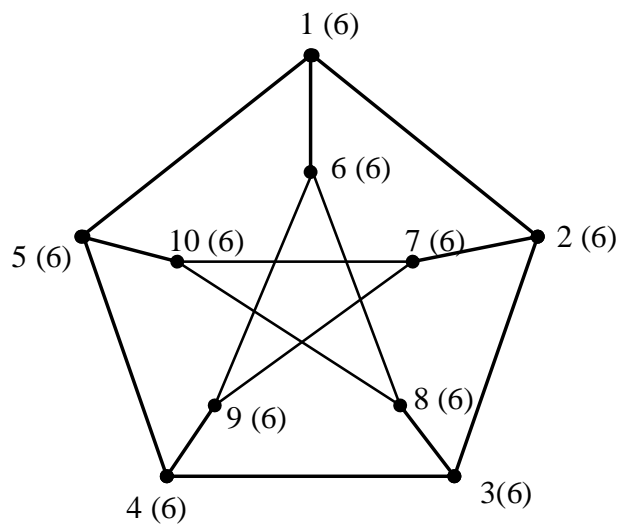

Figure 10: DRI of vertices of Petersen Graph. 
\{

$$
\text { Length - -; }
$$

\}

\section{CONCLUSION}

Floyd's, all pair shortest path algorithm is used to get the distance matrix in $O\left(n^{3}\right)$ time. The algorithm to find DRI of a vertex takes $O\left(n^{3}\right)$ of time. We can also use Dijkstra's single source shortest path algorithm [8] to find the distance matrix. In this case Dijkstra's algorithm has to be repeated $\mathrm{n}$ times. The complexity remains the same i.e. $O\left(n^{3}\right)$. The complexity of this method can be further reduced by using other data structures like adjacency list, heaps, queues [2, 9, and 10].

A lot of theoretical work can be undertaken with respect to DRI. Certain class of graphs can be characterised through the DRI sequences. For example the DRI sequences $(1,0,0, \ldots, 0,1),(2,2, \ldots, 2)$ characterize paths and cycles respectively.

\section{REFERENCES}

[1] Anany Levitin, Introduction to the design and analysis of Algorithms, $2^{\text {nd }} \mathrm{Ed}, 2008$.
[2] Coremen. T. H, Leiserson, C. E. Rivest R. L, and C Stein, Introduction to Algorithms, $2^{\text {nd }}$ Ed. MIT press, Cambridge, MA, 2001.

[3] Data Structures, Algorithms, and Applications in C++, Sartaj Sahni. McGraw-Hill Education, 1998.

[4] Distance in graphs - Fred Buckley, Frank Harary, Addison-Wesley Pub. Co., ()1990 .

[5] Harary. F, Graph Theory, Addison Wesley.

[6] R.W. Floyd. Algorithm 97: Shortest path. Comm. ACM, 5:345, 1962.

[7] Tarjan, R. E. (1972), "Depth-first search and linear graph algorithms", SIAM Journal on Computing 1 (2): 146160.

[8] E. W. Dijkstra. A Note on Two Problems in Connexion with Graphs. Numer. Math., 1:269-271, 1959.

[9] A. V. Goldberg and C. Silverstein. Implementations of Dijkstra's Algorithm Based on Multi-Level Buckets. In P. M. Pardalos, D.W. Hearn, and W.W. Hages, editors, Lecture Notes in Economics and Mathematical System 450 (Refereed Proceedings), pages 292-327. Springer Verlag, 1997.

[10] Boris V. Cherkassky , Andrew V. Goldberg , Craig Silverstein, Buckets, Heaps, Lists, and Monotone Priority Queues, SIAM Journal on Computing, v.28 n.4, p.13261346, Aug. 1999. 